\title{
Calibration of a Physics-Based Model of an Anthropomimetic Robot using Evolution Strategies
}

\author{
Steffen Wittmeier, Andre Gaschler, Michael Jäntsch, Konstantinos Dalamagkidis and Alois Knoll
}

\begin{abstract}
The control of tendon-driven and, in particular, of anthropomimetic robots using techniques from traditional robotics remains a very challenging task $[1,2]$. Hence, we previously proposed to employ physics-based simulation engines to simulate the complex dynamics of this emerging class of robots [3] and to use the simulation model as an internal model for robot control [4]. This approach, however, relies on an accurate model to be successful.

In this paper, we present the automated, steady-state pose calibration of a physics-based, anthropomimetic robot model using a $(\mu, \lambda)$-Evolution Strategy. For the acquisition of the poses of the physical robot, a stereo-vision, infrared-marker based motion capture system with real-time capabilities was developed. The employed $(\mu, \lambda)$-Evolution Strategy uses a Gaussian-based, non-isotropic, self-adapting mutation operator to explore the search space and reduce the simulation-reality gap. The obtained results are impressive, resulting in a reduction of joint angle errors in the range of one to two orders of magnitude and an absolute joint angle error of $0.5^{\circ}-4.5^{\circ}$ per pose evaluated.
\end{abstract}

\section{INTRODUCTION}

In robotics, computer simulations have become an important tool that is mainly used offline for controller design and optimization as a cheap and safe substitute of the real hardware [5]. But online applications are also suggested, such as an internal model for functional imagination [6]. These online applications, however, impose very specific requirements on the simulation software such as a real-time interface for updating and querying model parameters during simulation and, most importantly, rely on an accurate model of the simulated robot. For traditional robots, such a model can be derived analytically using either the Newton-Euler or Lagrangian formulation. For anthropomimetic [7] or musculoskeletal robots, however, a similar approach is not feasible due to the sheer complexity of the musculoskeletal structure which typically features non-revolute joints, muscles with pose-dependent lever arms, muscle vs. skeleton collisions and even multi-articular muscles. Therefore, the precise control of this emerging class of tendon-driven robots using techniques from traditional robotics remains a very challenging task that has been so far only successfully achieved for small-scale setups comprising exclusively revolute joints and low muscle redundancies $[1,2]$. To tackle this problem we previously proposed to use physics-based simulation engines to simulate the complex dynamics of anthropomimetic robots and to

The authors are with the Chair of Robotics and Embedded Systems, Faculty of Informatics, Technische Universität München, $\mathrm{Mu}$ nich, Germany; Correspondence should be addressed to $\mathrm{S}$. Wittmeier (steffen.wittmeierdin.tum.de)

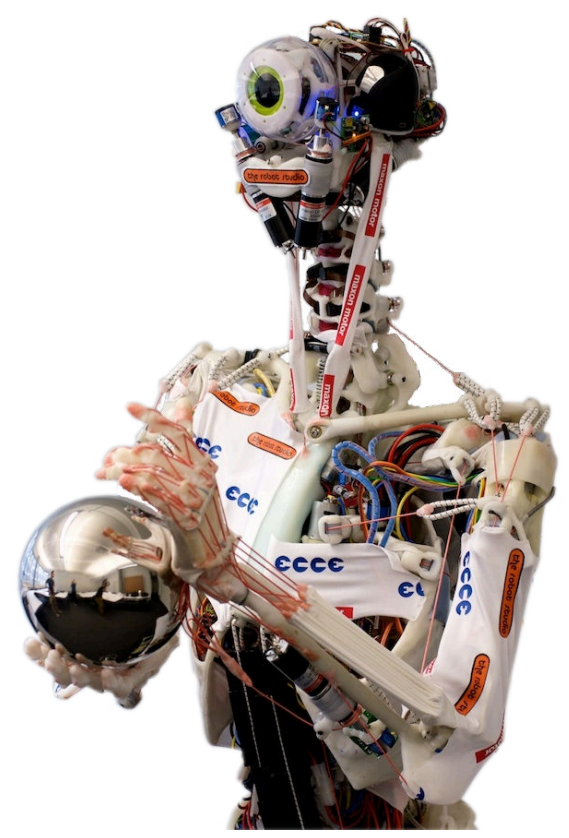

Fig. 1. Eccerobot Design Study (EDS). The EDS is an anthropomimetic robot [7] developed within the EU-funded project ECCEROBOT [8, 9]. The skeleton is hand-crafted using polymorph-a caprolactone polymer-which can easily be molded. The human muscles are imitated by elastic, tendondriven actuators.

employ the physics-based model as an internal model for control [3].

We already presented the derivation and implementation of the physics-based model of an anthropomimetic robot using the simulation framework CALIPER [3, 10]. However, even though the derived models of the individual sub-components of the robot, such as motors or springs, were validated against their real counterparts, preliminary simulations of the complete robot exhibited a significant simulation-reality gap. We hypothesize that this is not due to the physics-based simulation approach per se, but rather due to: (i) the handcrafted nature of the robot which makes it difficult to derive an accurate skeleton model and (ii) the modeling simplifications that were necessary in [3] to simulate the complex dynamics of the tendon-driven muscles. To evaluate this hypothesis, we now propose to use Machine Learning techniques, namely Evolution Strategies (ES), to calibrate the model and minimize the simulation-reality gap.

The physics-based model of the anthropomimetic robot 
(a) Ecce-IB

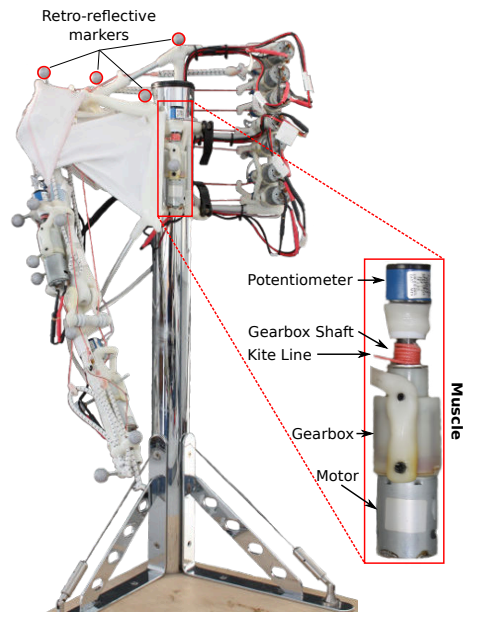

Fig. 2. Ecce-IB and Ecce-IB simulation model. a: The Ecce-IB comprises a spherical shoulder and a revolute elbow joint as well as 11 tendon-driven muscles. b: Physics-based simulation model of ECCE-IB [3] rendered by the tendon-driven robot simulation framework CALIPER [10].

described in [3] consisted of $\sim 300$ parameters which impedes a simultaneous calibration. Hence, for the first experiments, we decided to focus on a subset of $\sim 100$ parameters by limiting the calibration routine to steady-state poses and model parameters that could not be identified accurately in [3]. Once a satisfactory calibration of these steady-state parameters is achieved, we will investigate the feasibility of extending the ES approach to the calibration of the system dynamics. For the calibration, we recorded synchronized sensor data of various steady-state poses of the robot (gearbox shaft positions of all muscles and corresponding joint angles) and evaluated the individuals of the ES by setting the recorded gearbox shaft positions as control reference and using the resulting joint angle deviations as fitness measure. After calibration, we validated the quality of the calibration by analyzing the joint errors of the model for steady-state poses that were not part of the previous calibration phase. If the ES approach proves to be successful, the calibrated, physics-based model will serve as: (i) a virtual test-bed for novel control strategies and (ii) as an internal model of the robot to be used online for control.

The anthropomimetic robot platform and the implemented Evolution Strategy are introduced in Section II and III, respectively. Section IV describes the developed stereo-vision motion capture system used to acquire the joint angles of the steady-state robot poses whereas Section $\mathrm{V}$ presents the obtained calibration and validation results. Finally, conclusions and future work prospects are summarized in Section VI.

\section{The Anthropomimetic Robot Platform}

There are many humanoid robotic projects being undertaken around the world. However, most of them mainly focus on adopting the morphological appearance of humans and employ sophisticated actuation and control techniques which results in an impressive performance for the tasks that they are designed for $[11,12]$. However, these robots are still far from capturing the distinct dynamics and impressive adaptability to changing environments that humans possess. Hence, the EU-funded project ECCEROBOT takes a fundamentally different, namely anthropomimetic [7], approach. Instead of only imitating the morphological appearance, it tries to also mimic the internal structures of humans: bones, joints, muscles and tendons.

Currently, four ECCEROBOTS exist (listed in chronological order): (i) the ECCE-IA [8], (ii) the ECCE-IB (see Fig. 2a), (iii) the Eccerobot Design Study (EDS) (see Fig. 1 and [8]) and (iv) the ECCE-II. The skeletons of all prototypes are handcrafted using polymorph - a caprolactone polymer-which can easily be molded at a temperature of only $60^{\circ} \mathrm{C}$. As muscle equivalents, compliant tendon-driven actuators are used that consist of a DC motor and a gearbox in series with a kite line and a shock cord that function as the muscle fibers and tendon (see Fig. 2a and Fig. 2 in [3]). By coiling the kite line around the gearbox shaft, the artificial muscle can either be innervated or relaxed depending on the direction of rotation of the shaft. To simplify the muscle apparatus, only the most important of the $\sim 650$ human muscles are replicated and the attachment points are chosen accordingly to resemble humanlike movement dynamics.

The ECCE-IA, EDS and ECCE-II torsos each consist of more than 40 powered degrees of freedom, which complicates the analysis of the overall system dynamics. Hence, the ECCE-IB, which was used for the results presented in this work, has been manufactured by the ECCEROBOT consortium to reduce the complexity of the first experiments. In contrast to the full torsos, ECCE-IB features only one arm. However, the key properties of the anthropomimetic approach are still preserved. ECCE-IB contains a spherical shoulder and a revolute elbow joint, as well as 11 powered degrees of freedomtwo for the elbow, eight for the shoulder and one bi-articular muscle affecting both shoulder and elbow joint angles. For proprioception-the sense of static positions and movements of the limbs of the body [13] — each artificial ECCE-IB muscle is equipped with: (i) a potentiometer to measure the position of the gearbox shaft and (ii) a current sensor within the electronic control units (ECUs) for DC motor control [14].

\section{Evolution Strategy}

As ES, a comma selection variant of the form $(\mu, \lambda)$ was chosen, where $\mu$ and $\lambda$ are the population sizes of the parent and offspring populations, respectively. In contrast to plus selection $(\mu+\lambda)$, the comma selection uses only the offspring population as selection pool and has been shown to be preferred over the plus variant for unbounded, real-valued search spaces [15]. The parent and offspring populations $\left(P_{p}\right.$ and $P_{o}$, respectively) consist of individuals $I$ of the form:

$$
I_{k}=\left(\vec{y}_{k}, \vec{s}_{k}, F\left(\vec{y}_{k}\right)\right)
$$

where $\vec{y}_{k}$ are the model object parameters modified by the mutation, $\vec{s}_{k}$ are the evolving endogenous strategy parameters defining the step-size of the mutation and $F\left(\vec{y}_{k}\right)$ is the real-valued fitness of individual $k$. The used ES algorithm 


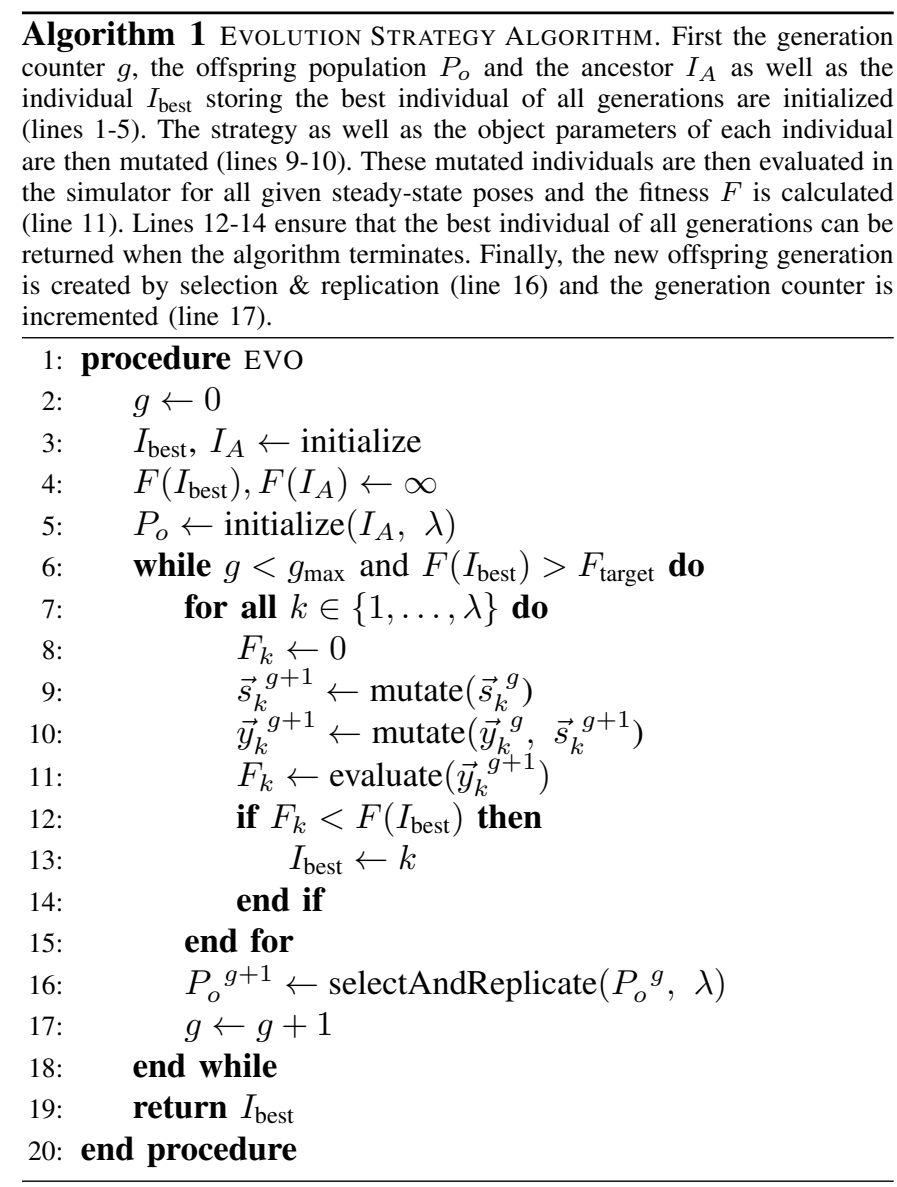

is summarized in Algorithm 1 and the employed mutation, evaluation, and selection/replication operations as well as the termination conditions are described in the following subsections.

\section{A. Mutation}

For mutation, a Gaussian-based, non-isotropic mutation operator with strategy parameter self-adaptation was used [16]. To ensure that the mutated object parameter values do not exceed a specified, reasonable value range (e.g. spring constants have to be positive), positive and negative value range limits can be defined for each object parameter. If the mutated object parameter value exceeded either one of the limits, it was set to the limiting value.

\section{B. Evaluation}

During evaluation, each individual $k$ of the offspring population $P_{o}$ was evaluated using $p$ steady-state poses $\vec{S}$ of the form:

$$
\vec{S}=\left[\begin{array}{ll}
\vec{G}^{T} & \vec{q}^{T}
\end{array}\right]
$$

where $\vec{G}$ are the gearbox shaft positions of the $m$ robot muscles and $\vec{q}$ are the angles of the $n$ robot joints. The evaluation of each steady-state pose $\vec{S}$ proceeded as follows: First $\vec{G}$ was set as references for the gearbox shaft position controllers of the simulated muscles (see [3]) and the simulation was stepped for the pose-acquisition time $t_{a}$. Following each simulation step, the gearbox shaft position error vector $\triangle \vec{G}$ was computed from the current and reference positions, respectively. If $t_{a}$ was reached and $\max (\triangle \vec{G})$ was greater than a user-defined $\triangle G_{\max }$, the individual was marked as invalid and the evaluation of this individual was aborted. Otherwise, the simulation was stepped for an additional pose-settle time $t_{s}$ and the joint angular velocities $\vec{\omega}$ were computed after each step. Only if $\max (\vec{\omega})$ was less than a user-defined $\omega_{\max }$ for the steady-state duration $t_{d}$ the model was considered to be in equilibrium. However, if this condition was not satisfied before $t_{s}$ was reached, the individual was marked as invalid and the evaluation of this individual was aborted. Otherwise, the simulation model was reloaded to guarantee identical initial conditions for the evaluation of all poses and the evaluation proceeded with the next pose $\vec{S}$. If the individual was not marked as invalid after the evaluation of all $p$ poses, the fitness of the individual was computed as follows:

$$
F\left(\vec{y}_{k}\right)=\sum_{i=1}^{p} \sum_{j=1}^{n} \alpha_{i, j}^{2}
$$

where $\alpha_{i, j}$ is the joint angle error between the robot and the simulation for joint $j$ and steady-pose $i$, respectively. For revolute joints, the joint angles $q$ and $q^{\prime}$ of the robot and the simulation, respectively, are scalars and the error $\alpha_{i, j}$ can easily be computed. However, in the case of spherical joints, the joint angles were expressed using the quaternions $Q$ and $Q^{\prime}$ and $\alpha_{i, j}$ was computed using the quaternion distance defined as:

$$
\alpha_{i, j}=\arccos \left(Q_{i, j} \cdot Q_{i, j}^{\prime}\right)
$$

\section{Selection \& Replication}

The best $\mu$ valid individuals of $P_{o} g$ were selected and replicated to form the offspring population of the next generation $P_{o}{ }^{g+1}$. If no single valid individual existed, the algorithm terminated. As comma selection strategies use only the offspring population as selection pool, the best individual found so far does not survive (in contrast to plus selections). Hence, an additional individual $I_{\text {best }}$ was introduced that stores the best individual of all generations and that was returned when the algorithm terminated.

\section{Termination}

As termination condition the following resource and convergence criteria were used:

$$
\begin{array}{r}
g \geq g_{\max } \\
F\left(I_{\text {best }}\right)<F_{\text {target }}
\end{array}
$$

where $g$ and $g_{\max }$ are the current and maximum number of generations, respectively, and $F_{\text {target }}$ is a user-defined target fitness. 


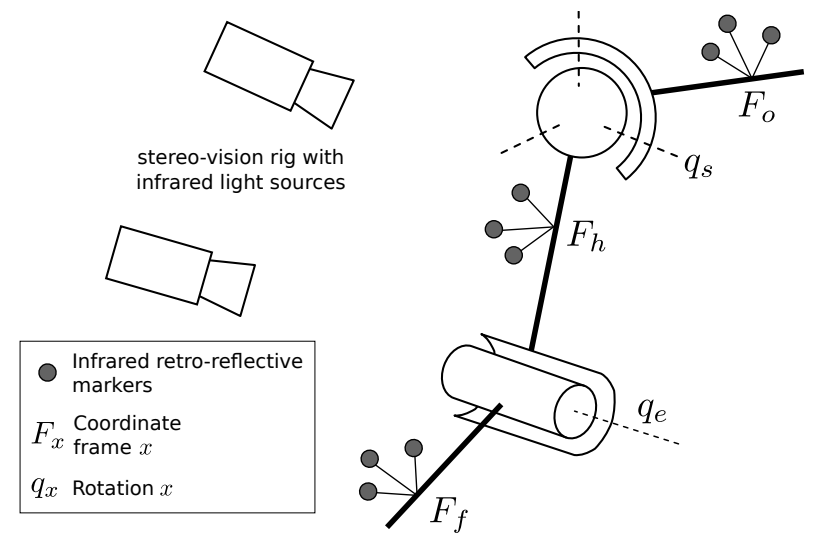

Fig. 3. Motion Capture System. A stereo-vision motion capture system built from commodity hardware was developed to provide the joint angles of the steady-state poses that are required as input data for the ES algorithm. The motion capture system uses infrared retro-reflective markers attached to the limbs of the robot that are illuminated by infrared light sources.

\section{Motion CApture System}

In humans, mechanoreceptors in muscles and joints provide the data for the two submodalities of proprioception: (i) limb positioning and (ii) limb movement [13]. For limb positioning - the task we try to achieve in this paper-, the joint capsule mechanoreceptors are particularly important as they provide a feedback measure for the orientation of a joint. However, developing and integrating similar joint angle sensors for non-revolute or non-prismatic joints, such as the spherical joints of the ЕсСЕRовотS is an inherently difficult task (see also [17]) and hence, the ЕССЕROBOTs are not equipped with analogous intrinsic sensors. Thus, it was necessary to employ an extrinsic sensing system to provide the input data required by the ES.

For that purpose, we developed a dedicated, stereo-vision motion capture system capable of providing joint angle data in real-time based on [18]. The system uses PointGrey Flea2 cameras with Pentax $6 \mathrm{~mm}$ optics equipped with infrared pass filters $\left(\lambda_{\text {thresh }}=750 \mathrm{~nm}\right)$ and spherical, retro-reflective markers attached to the robot limbs that are illuminated by infrared LED clusters positioned next to the cameras $\left(\lambda_{\text {LED }}=880 \mathrm{~nm}\right.$, see Figs. 2A and 3). The acquired stereo images were processed by conventional image segmentation and 3D triangulation methods in order to obtain the set of 3D marker positions. Subsequently, the combined matching and pose recovery problem of the rigid marker targets was solved to estimate the marker frames $F_{o}, F_{h}$ and $F_{f}$, respectively (see Fig. 3). Here, a custom graph-based algorithm has been implemented that exploited a-priori knowledge about the marker targets to minimize the computational effort while, at the same time, ensured a high fitting accuracy (a root mean squareRMS—re-projection error of $<0.3 \mathrm{~mm}$ was achieved). In the final processing step, the joint orientations of the shoulder and elbow joint ( $q_{s}$ and $q_{e}$, respectively) were computed from the corresponding marker frame pairs $\left(\left\{F_{o}, F_{h}\right\}\right.$ and $\left.\left\{F_{h}, F_{f}\right\}\right)$.

The motion capture system was implemented using a shared memory based software pipeline toolkit (QPT [19]) to minimize the pipeline latency and integrated into the ЕсСЕRовот software framework to obtain joint angle data synchronized with other sensor modalities.

\section{OUR APPROACH \& RESUlts}

We hypothesize that the simulation-reality gap observed during preliminary simulations of the complete ECCE-IB model is mainly due to the three following muscle model simplifications that were made in [3]: (i) the passive elements of the simulated muscle (kite line and shock cord) are not represented as a collision entity within the simulation and hence, no collisions between the muscles and the skeleton are simulated, (ii) the muscle attachment points were approximated based on laser-scan data and (iii) the muscles were simulated as direct connection between the two attachment points $\vec{A}_{1}$ and $\vec{A}_{2}$ whereas the ECCE-IB muscles often wrap around the skeleton. All three simplifications have a high impact on the applied muscle forces and hence on the statics and dynamics of the model. To corroborate this hypothesis, we started with a minimal object parameter set for the ES which comprised only muscle parameters. For each of the 11 robot muscles, we therefore selected the following object parameters: (i) the muscle attachment points $\vec{A}_{1}$ and $\overrightarrow{A_{2}}$ (both $\in \mathbb{R}^{3}$ ), (ii) the initial kite line length $L_{K_{0}}$ which is required for the calibration of the potentiometer sensor model used for the gearbox shaft controller (see [3]), (iii) the spring constant of the muscle flexibility, and (iv) the gearbox shaft spindle radius which is considered to be constant in the simulation but varies stochastically in reality due to the coiled kite line. These 99 object parameters were initialized for the ancestor individual $I_{A}$ based on laser scan data and manual measurements.

To test the ES calibration algorithm and to identify strategy parameter step-sizes that provide a high convergence rate, we first calibrated the model using three elbow and a fixed shoulder pose (see Section V-A). Subsequently, we calibrated the entire joint space of the robot arm using 15 calibration poses (see Section V-B). In both cases, the best individual obtained from the calibration was validated using a second, disjunct set of steady-state poses. In addition to the fitness, we introduced a mean joint error (MJE) to quantify the accuracy of the calibration and validation results which was defined as:

$$
\operatorname{MJE}_{j}=\left(\sum_{i=1}^{p}\left|\alpha_{i, j}\right|\right) / p, \text { with } j \in\{e, s\}
$$

\section{A. Elbow Calibration \& Validation}

The elbow joint of the simulation model presented in [3] was calibrated using three steady-state poses that covered a wide range of elbow joint movements while the shoulder joint angle was kept constant. As anthropomimetic robots do not provide the repeatability of industrial manipulators, each pose was acquired 5 times to compute the mean and standard deviation of the joint angles that were subsequently used as input data for the ES $\left(\bar{q}_{1}=0^{\circ}, \bar{\sigma}_{1}=0.26^{\circ}\right.$; $\left.\bar{q}_{2}=58^{\circ}, \bar{\sigma}_{2}=0.31^{\circ} ; \bar{q}_{3}=110^{\circ}, \bar{\sigma}_{3}=0.91^{\circ}\right)$. The 
(a) Mean Joint Error \& Fitness

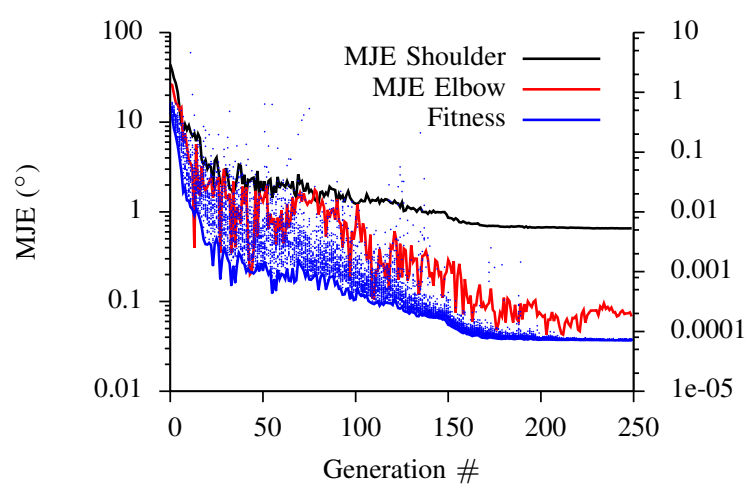

(b) Pose Errors

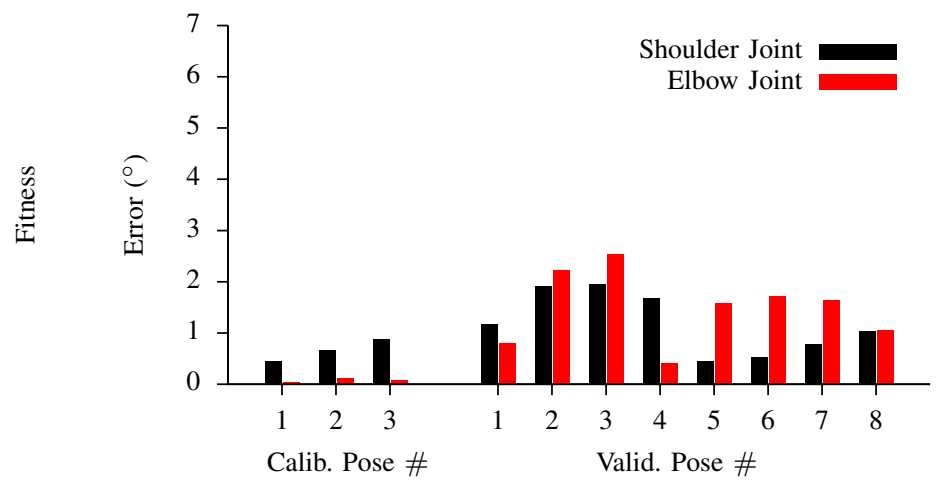

Fig. 4. Elbow Calibration \& Validation Results. a: Convergence of mean joint error (MJE) and fitness for the calibration of the three elbow calibration poses shown in (b). The poses covered an elbow movement range of $110^{\circ}$ and a final $\mathrm{MJE}$ of $0.08^{\circ}$ and $0.66^{\circ}$ was achieved after 250 generations for the elbow and shoulder joint, respectively. b: Calibration and validation pose errors of $I_{\text {best }}$ for the three calibration and 8 validation poses. For the validation, a MJE $e$ and $\mathrm{MJE}_{s}$ of $1.1^{\circ}$ and $1.0^{\circ}$ was achieved.

parent $P_{p}$ and offspring $P_{o}$ population sizes were set to $\mu=6$ and $\lambda=36$, respectively, to achieve a $\mu / \lambda$-ratio of $1 / 6$ which has been shown to provide a maximum rate of convergence [20]. The maximum number of generations was limited to 250 and the maximum joint velocity and gearbox shaft position error were parametrized to be $\omega_{\max }=0.02 \mathrm{rad} / \mathrm{s}$ and $\triangle G_{\max }=5^{\circ}$, respectively. A sequence of calibration runs was performed to determine initial strategy parameter stepsizes that provided a high convergence rate and the following settings were eventually identified: $25 \mathrm{~N} \mathrm{~m}^{-1}$ for the spring constants, $5 \cdot 10^{-5} \mathrm{~m}$ for the spindle radii and $2 \cdot 10^{-3} \mathrm{~m}$ for both, the muscle attachment points and $L_{K_{0}}$. Furthermore, a value range of $\left[1 \mathrm{~N} \mathrm{~m}^{-1} 10^{4} \mathrm{~N} \mathrm{~m}^{-1}\right]$ and $\left[5 \cdot 10^{-3} \mathrm{~m} 10^{-2} \mathrm{~m}\right]$ was defined for the spring constants and spindle radii to ensure reasonable values after mutation. $t_{m}$ and $t_{s}$ were set to $5 \mathrm{~s}$, whereas the steady-state duration $t_{d}$ was configured to be $0.5 \mathrm{~s}$.

The convergence of the elbow and shoulder mean joint error $\left(\mathrm{MJE}_{e}\right.$ and $\mathrm{MJE}_{s}$, respectively) as well as of the fitness for these settings is shown in Fig. 4a. At $g=0, \mathrm{MJE}_{e}$ was equal to $26.6^{\circ}$ and $\mathrm{MJE}_{s}$ was approximately $44^{\circ}$. However, the ES converged rapidly and after 27 generations, the MJE of the elbow was already reduced to the sub-degree domain. These intermediate results were further improved and a final MJE of $0.08^{\circ}$ and $0.66^{\circ}$ was reached after 250 generations for the elbow and shoulder joint, respectively (see Fig. 4b).

The calibrated results were assessed using an additional, disjunct set of 8 elbow poses (derived by linear interpolation of the gearbox shaft reference positions for two neighboring calibration poses). The results of this validation step are also shown in Fig. 4b. Again, it can be seen that the average error was successfully minimized, varying in the range of $0.4^{\circ}$ to $2.5^{\circ}$ dependent on the pose and joint, respectively.

\section{B. Robot Calibration \& Validation}

We then calibrated the entire joint space of the robot using 15 calibration poses. The ES settings were identical to the elbow calibration (see previous section) and the calibration results were assessed using a second set of 15 validation poses.

The results of the calibration and validation are shown in Fig. 5. At $g=0$, the mean joint errors of the elbow and shoulder joint are equal to $\mathrm{MJE}_{e}=32.9^{\circ}$ and $\mathrm{MJE}_{s}=35.7^{\circ}$, respectively. But, similar to the previous calibration, the ES converged quickly and an elbow and shoulder MJE of $3.2^{\circ}$ and $4.8^{\circ}$ was reached after 42 generations. However, these intermediate results were further optimized and a final $\mathrm{MJE}_{e}$ and $\mathrm{MJE}_{s}$ of $1.9^{\circ}$ and $2.0^{\circ}$ was eventually achieved after the full run of 250 generations which took $\sim 45 \mathrm{~h}$ on an Intel i7 desktop computer.

\section{CONCLUSIONS AND Future WORKS}

\section{A. Conclusions}

We presented the steady-state pose calibration of a physicsbased model of an anthropomimetic robot using a $(\mu, \lambda)$ Evolution Strategy. To compensate for the lack of joint angle sensors, a stereo-vision based motion capture system was developed to obtain the input data required by the ES as well as to quantify the simulation-reality gap. The calibration procedure was performed in two steps. First, a sub-domain of the joint space was calibrated to determine strategy parameter settings that provided a high convergence rate. Subsequently, the entire joint space of the robot was calibrated using a total of 15 steady-state poses. All calibration results were assessed using additional, disjunct sets of validation poses. Even though a simplified muscle model was used to simulate the dynamics of the musculoskeletal structure, the ES converged quickly and a final average joint angle error of $1.9^{\circ}$ and $2.0^{\circ}$ was achieved for the elbow and shoulder joint, respectively. This demonstrates that the simplified muscle model, as presented in [3], is sufficient to approximate the tendon-driven muscular system of this anthropomimetic robot. However, whether the ES approach is superior to other calibration techniques for real-valued search-spaces can not be inferred from the results and additional tests with alternative algorithms would be required. But, our goal was to minimize the simulation-reality 
(a) Mean Joint Error \& Fitness

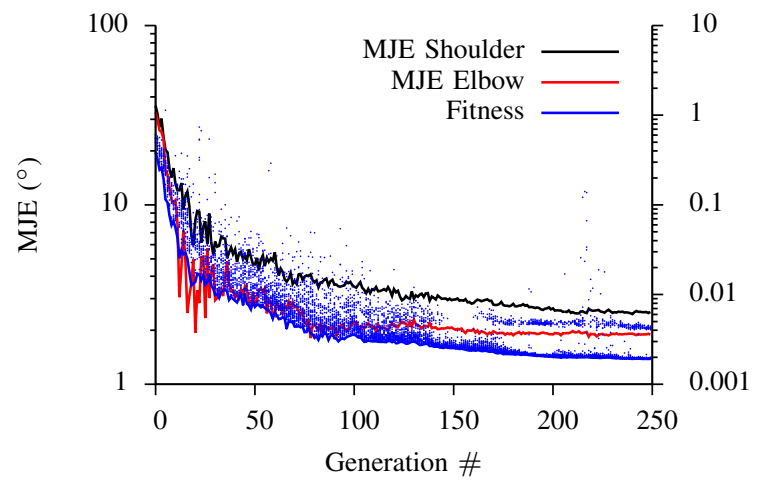

(b) Pose Errors

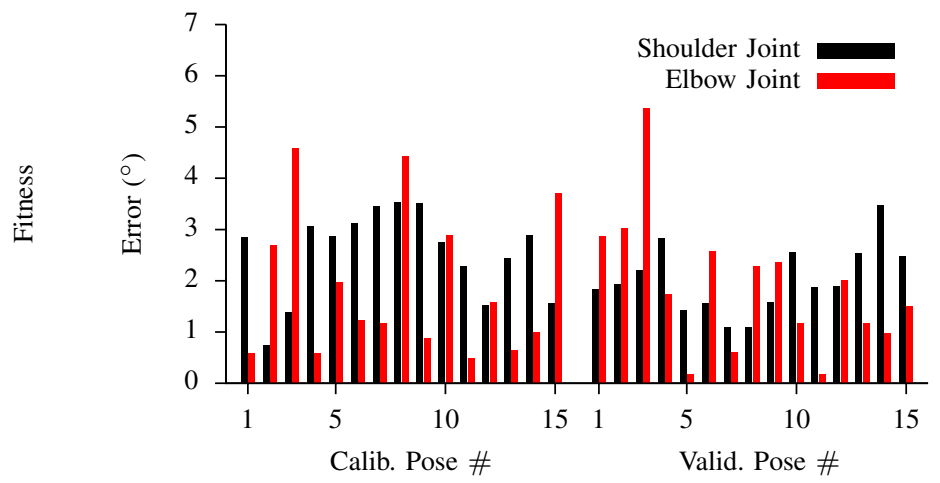

Fig. 5. Robot Calibration \& Validation Results. a: Convergence of the mean joint error (MJE) and fitness for the calibration of the 15 calibration poses shown in (b). At generation 0, the mean joint error of the elbow and shoulder joint were equal to $32.9^{\circ}$ and $35.7^{\circ}$, respectively. However, th ES converged quickly, and a final $\mathrm{MJE}_{e}$ and $\mathrm{MJE}_{s}$ of $1.9^{\circ}$ and $2.5^{\circ}$ were reached for the best individual $I_{\text {best }}$ after 250 generations. b: Calibration and validation pose errors of $I_{\text {best }}$ for the 15 calibration and validation poses. For the validation, a $\mathrm{MJE}_{e}$ and $\mathrm{MJE}_{s}$ of $1.9^{\circ}$ and $2.0^{\circ}$ was achieved.

gap in order to use the model for the software-in-the-loop testing of controllers and as an internal model for control and in that we succeeded.

\section{B. Future Works}

In the future we will investigate the possibility of extending the ES approach to the calibration of the system dynamics. The presented motion capture system has already been developed with that in mind and is able to provide the required joint angle data in real-time. Furthermore, we will test the ES approach to calibrate a model of a full anthropomimetic torso. But this should be possible as the task of calibrating the entire torso can be decomposed into multiple sub-tasks of similar complexity as the presented results (e.g. calibration of left arm, right arm, etc.). The calibrated physics-based model will finally be employed as a software-in-the-loop tool for the development and testing of novel control strategies as well as an internal model for robot control.

\section{ACKNOWLEDGEMENTS}

The authors thank F. Sehnke for his valuable advice related to Evolution Strategies. The research leading to these results has received funding from the European Community's Seventh Framework Programme FP7/2007-2013 Challenge 2- Cognitive Systems, Interaction, Robotics - under grant agreement no. 231864 - ECCEROBOT

\section{REFERENCES}

[1] H. Kino, S. Kikuchi, T. Yahiro, and K. Tahara, "Basic study of biarticular muscle's effect on muscular internal force control based on physiological hypotheses," in Proc. IEEE International Conference on Robotics and Automation ICRA '09, 2009, pp. 4195-4200.

[2] S. C. Jacobsen, H. Ko, E. K. Iversen, and C. C. Davis, "Antagonistic control of a tendon driven manipulator," in Proc. Conf. IEEE Int Robotics and Automation, 1989, pp. 1334-1339.

[3] S. Wittmeier, M. Jäntsch, K. Dalamagkidis, and A. Knoll, "Physicsbased Modeling of an Anthropomimetic Robot," in Proc. IEEE/RSJ International Conference on Intelligent Robots and Systems (IROS), 2011, accepted.

[4] M. Kawato, "Internal models for motor control and trajectory planning," Current Opinion in Neurobiology, vol. 9, no. 6, pp. 718-727, 1999

[5] J.-J. E. Slotine and W. Li, "On the Adaptive Control of Robot Manipulators," The International Journal of Robotics Research, vol. 6, no. 3, pp. 49-59, 1987.
[6] H. G. Marques and O. Holland, "Architectures for functional imagination," Neurocomput., vol. 72, no. 4-6, pp. 743-759, 2009.

[7] O. Holland and R. Knight, "The anthropomimetic principle," in Proceedings of the AISB06 Symposium on Biologically Inspired Robotics, 2006.

[8] H. Marques, M. Jäntsch, S. Wittmeier, O. Holland, C. Alessandro, A. Diamond, M. Lungarella, and R. Knight, "ECCE1: The first of a series of anthropomimetic musculoskeletal upper torsos," in 10th IEEERAS International Conference on Humanoid Robots (Humanoids), Dec 2010, pp. $391-396$.

[9] Embodied Cognition In A Compliantly Engineered Robot (ECCEROBOT). [Online]. Available: http://www.eccerobot.eu

[10] S. Wittmeier, M. Jäntsch, K. Dalamagkidis, M. Rickert, H. G. Marques, and A. Knoll, "Caliper: A Universal Robot Simulation Framework for Tendon-Driven Robots," in Proc. IEEE/RSJ International Conference on Intelligent Robots and Systems (IROS), 2011, accepted.

[11] Y. Sakagami, R. Watanabe, C. Aoyama, S. Matsunaga, N. Higaki, and K. Fujimura, "The intelligent asimo: system overview and integration," in Intelligent Robots and Systems, 2002. IEEE/RSJ International Conference on, vol. 3, 2002, pp. $2478-2483$ vol.3.

[12] K. Kaneko, K. Harada, F. Kanehiro, G. Miyamori, and K. Akachi, "Humanoid robot hrp-3," in Intelligent Robots and Systems, 2008. IROS 2008. IEEE/RSJ International Conference on, Sep 2008, pp. 2471 -2478.

[13] E. R. Kandel, J. Schwartz, and T. M. Jessell, Principles of Neural Science, 4th ed. Mc Graw Hill, 2000.

[14] M. Jäntsch, S. Wittmeier, and A. Knoll, "Distributed Control for an Anthropomimetic Robot," in Intelligent Robots and Systems (IROS), 2010 IEEE/RSJ International Conference on, Oct 2010, pp. 5466 -5471.

[15] H. Schwefel, "Collective Phenomena in Evolutionary Systems," in Preprints of the 31st Annual Meeting of the International Society for General System Research, 1987, pp. 1025-1033.

[16] H.-G. Beyer and H.-P. Schwefel, "Evolution strategies - a comprehensive introduction," Natural Computing, vol. 1, pp. 352, May 2002.

[17] J. Urata, Y. Nakanishi, A. Miyadera, I. Mizuuchi, T. Yoshikai, and M. Inaba, "A Three-Dimensional Angle Sensor for a Spherical Joint Using a Micro Camera," in Proc. of the IEEE International Conference on Robotics and Automation, 2006, pp. 4428-4430.

[18] A. Gaschler, "Visual motion capturing for kinematic model estimation of a humanoid robot," in Proceedings of the 33rd international conference on Pattern recognition. Springer, 2011, pp. 438-443.

[19] Qt Pipeline Toolkit (QPT). S. Wittmeier. [Online]. Available: http://sourceforge.net/p/qpt

[20] F. Hoffmeister and T. Bäck, "Genetic algorithms and evolution strategies - similarities and differences," in Proceedings of the 1st Workshop on Parallel Problem Solving from Nature, ser. PPSN I. London, UK: Springer-Verlag, 1991, pp. 455-469. 